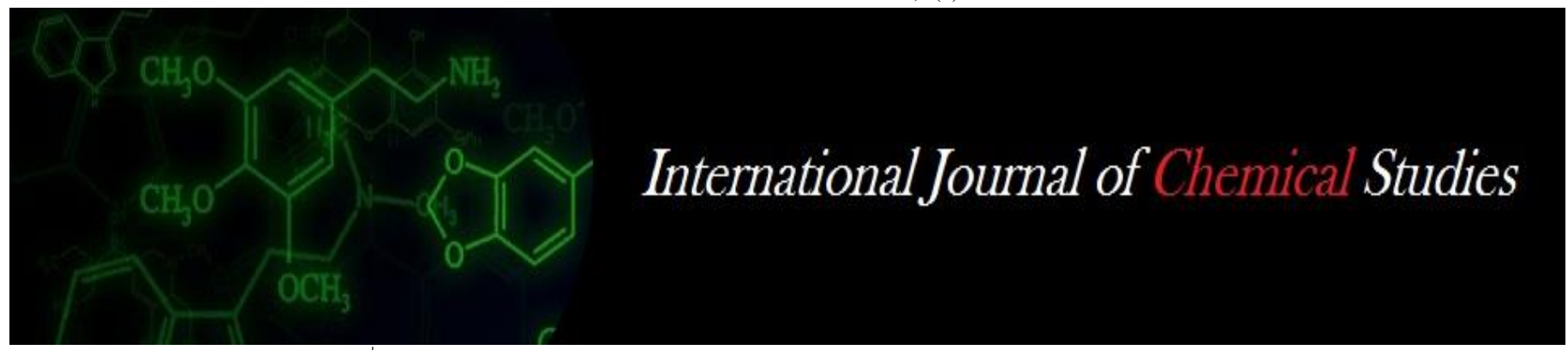

P-ISSN: 2349-8528

E-ISSN: 2321-4902

www.chemijournal.com

IJCS 2021; 9(1): 1663-1668

(C) 2021 IJCS

Received: 19-10-2020

Accepted: 23-11-2020

\section{SN Banne}

Department of Plant Pathology, College of Agriculture, Latur, Vasantrao Naik Marathwada Krishi Vidyapeeth, Parbhani, Maharashtra, India

\section{Sunita J Magar}

Department of Plant Pathology College of Agriculture, Latur, Vasantrao Naik Marathwada Krishi Vidyapeeth, Parbhani, Maharashtra, India

\section{Shruti S Kadam}

Department of Plant Pathology, College of Agriculture, Latur, Vasantrao Naik Marathwada Krishi Vidyapeeth, Parbhani,

Maharashtra, India

\section{AS Shinde}

Department of Plant Pathology, College of Agriculture, Latur, Vasantrao Naik Marathwada Krishi Vidyapeeth, Parbhani, Maharashtra, India
Corresponding Author:

\section{SN Banne}

Department of Plant Pathology, College of Agriculture, Latur, Vasantrao Naik Marathwada Krishi Vidyapeeth, Parbhani, Maharashtra, India

\section{In vitro efficacy of fungicides against Alternaria alternata (Fr.) Keissler, causing Leaf Blight disease of Chrysanthemum}

\author{
SN Banne, Sunita J Magar, Shruti S Kadam and AS Shinde
}

DOI: https://doi.org/10.22271/chemi.2021.v9.i1x.11464

\begin{abstract}
Chrysanthemum \{Dendranthema indicum (= Chrysanthemum indicum L.) \}, crop is being affected by several fungal, bacterial, viral and nematode induced diseases. However, leaf blight, caused by Alternaria alternata (Fr.) Keissler has been common occurrence, causing quantitative as well as qualitative losses in chrysanthemum. Therefore, present in vitro study was planned in CRD with two separate experiments (Contact and systemic and combi-fungicides) in vitro, in 13 treatments replicated thrice, to assess the efficacy of fungicides against Alternaria alternata, at the Department of Plant Pathology, College of Agriculture, Latur (MS), during Rabi, 2019-20. Results revealed that, all of the six systemic (each @ 500, 1000 and $1500 \mathrm{ppm}$ ), five contact and two combi-products fungicides (each @ 1000, 2000 and 2500 $\mathrm{ppm}$ ) evaluated in vitro were found fungistatic and exhibited significant mycelial growth inhibition of $A$. alternata, causing Alternaria leaf blight of chrysanthemum. However, the systemic fungicides viz., Propiconazole 25\% EC and Hexaconazole 5\% EC (100\%), followed by Difenconazole 25\% EC $(87.81 \%)$, Penconazole $10 \%$ EC $(86.13 \%)$ were found most effective against the test pathogen. Whereas, among contact / combi - fungicides the most effective fungicide found were Carbendazim $12 \% \mathrm{WP}+$ Mancozeb 63\% WP with highest growth inhibition (81.96\%), followed by Mancozeb 75\% WP (78.05\%), Copperoxy chloride 50\% WP (69.36\%), respectively.
\end{abstract}

Keywords: Chrysanthemum, Alternaria alternata, fungicides, inhibition

\section{Introduction}

Chrysanthemum (Chrysanthemum indicum L.) occupies a key position in the floriculture industry and it's the world's second most important floricultural crop after Rose (Kalia, 2015). It is also called as 'Queen of East' in European countries and commonly called as gul-e-daudi or golden flower autumn queen. Chrysanthemums belong to the Asteraceae (Compositae) family. It is the best dollar earning flower in United States; also grown in India and Maharashtra as a cut flower. In India, chrysanthemum commercially growing major states are Karnataka, Tamilnadu, Maharashtra, Rajasthan, Madhya Pradesh and Bihar. In Maharashtra, chrysanthemum is grown on an area of 0.39 thousand ha with the production of 1.65 thousand tonnes loose flowers and 0.05 thousand tonnes cut flowers (Anonymous, 2018) ${ }^{[1]}$. In Maharashtra, the leading districts in floriculture production are Nasik, Ahmednagar, Thane, Pune, Satara, Sangli and Nagpur. However, Ahmednagar district is specalized as growing district of the Maharashtra (Tupe et al., 2017) ${ }^{[18]}$.

Various biotic diseases are threatening the cultivation and good quality bloom yield of chrysanthemum. Among them, major disease are leaf blight (Alternaria alternata), leaf spot (Septoria chrysanthemella), wilts (Fusarium and Verticillium spp.), root rot (Pythium spp., Phytophthora spp.), powdery mildew (Golovinomyces chrysanthemi), dry root rot (Rhizoctonia solani), brown rust (Puccinia chrysanthemi), bacterial crown galls (Agrobacterium tumefaciens), bacterial blight (Pseudomonas cichori), viral stunt, mosaic and nematodes (Pradeepkumar et al., 2008) ${ }^{[14]}$. Among of these diseases, leaf blight caused by Alternaria alternata (Fries.) Keissler is one of the most destructive disease, commonly prevailing in almost all chrysanthemum growing areas and consequently causing accountable quantitative losses (> $80 \%$ yield losses) as well as deteriorating the quality of produce (Arunkumar, 2008; Divyajyothi et al., 2018) ${ }^{[3,7]}$. 
Therefore, present study on in vitro efficacy of fungicides against A. alternata, causing chrysanthemum Alternaria leaf blight was planned and conducted at the Department of Plant Pathology, College of Agriculture, Latur, during Rabi, 201920.

\section{Materials and Methods \\ Isolation, identification and pathogenicity of $\boldsymbol{A}$. alternata} Applying tissue isolation technique (Tuite, 1969) ${ }^{[19]}$, the fungus causing leaf blight disease in chrysanthemum was isolated from naturally infected chrysanthemum $(\mathrm{Cv}$. White probiotic) specimen, on autoclaved and cooled potato dextrose agar (PDA) plates. Pathogenicity test attempted by spray inoculating the spore suspension $\left(2 \times 10^{6}\right.$ spores $\left./ \mathrm{ml}\right)$ of the test pathogen, on chrysanthemum ( $\mathrm{Cv}$. White probiotic) seedlings in pot culture.

Based on typical symptoms of Alternaria blight (naturally and artificially diseased chrysanthemum plants), pathogenicity test, morpho-cultural characteristics and microscopic observations, the test pathogen was identified as Alternaria alternata (Fries.) Keissler., the cause of chrysanthemum Alternaria blight and further confirmed by comparing its authentic descriptions (Ellis, 1971) ${ }^{[8]}$.

In vitro evaluation of systemic fungicides: Two separate experiments were planned to evaluate in vitro efficacy of six systemic (each@ @ 500, 1000 and 1500 ppm) and five nonsystemic / contact plus two combi-products fungicides (each @ 1000, 2000 and 2500 ppm) and conducted with Completely Randomized Design (CRD) and all the treatments replicated thrice against A. alternata, causing leaf blight of chrysanthemum by applying Poisoned Food Technique (Nene and Thapliyal, 1993) ${ }^{[12]}$ and using Potato Dextrose Agar as a basal culture medium. Observations on radial mycelial growth / colony diameter $(\mathrm{mm})$ were in all the replicated treatments at $24 \mathrm{hrs}$ interval and continued till growth of the test pathogen in untreated control plates was fully covered. Per cent inhibition of the test pathogen over untreated control was calculated by applying following formula (Vincent, 1927).

$$
\begin{gathered}
\mathrm{C}-\mathrm{T} \\
\text { Per cent inhibition }=-------\times 100 \\
\mathrm{C}
\end{gathered}
$$

Where, $\mathrm{C}=$ Growth of the test fungus in untreated control plates

$\mathrm{T}=$ Growth of the test fungus in treated plates

The data obtained was statistically analyzed (Panse and Sukhatme, 1978) ${ }^{[13]}$ and the results were interpreted thereof.

\section{Result and Discussion}

The results obtained on per cent mycelial growth inhibition of test pathogen A. alternata, with the test systemic fungicides are depicted in PLATE-I and presented in Table 1 and Fig. 1. The test non systemic and combi- product fungicides are depicted in PLATE-II and presented in Table 2 and Fig. 2.

Mycelial growth inhibition of systemic fungicides: Results (PLATE I, Table 1, Fig. 1) revealed that, the systemic fungicides tested (each @ 500, 1000 and 1500 ppm) significantly inhibited mycelial growth of A. alternata, over untreated control and it was directly proportional to concentrations of the test fungicides. Results (PLATE I, Table 1, Fig. 1) revealed average mycelial growth inhibition recorded with the systemic fungicides ranged from 35.35 to 100.00 per cent. However, it was highest with Propiconazole $25 \%$ EC and Hexaconazole 5\% EC (100\%), followed by Difenconazole $25 \%$ EC $(87.81 \%)$, Penconazole $10 \%$ EC (86.13\%), Carbendazim 50\% WP $(47.82 \%)$ and Thiophanate methyl $70 \%$ WP $(35.35 \%)$, respectively, as against $(0.00) \mathrm{mm}$

\begin{tabular}{|c|c|c|c|c|c|c|c|c|c|}
\hline \multirow{2}{*}{$\begin{array}{l}\text { Tr. } \\
\text { No. }\end{array}$} & \multirow{2}{*}{ Treatments } & \multicolumn{3}{|c|}{ Col. Dia.* (mm) at ppm } & \multirow{2}{*}{ Av. $(\mathbf{m m})$} & \multicolumn{3}{|c|}{ \% Inhibition* at ppm } & \multirow{2}{*}{$\begin{array}{l}\text { Av. Inhib. } \\
(\%)\end{array}$} \\
\hline & & 500 & 1000 & 1500 & & 500 & 1000 & 1500 & \\
\hline $\mathrm{T}_{1}$ & Propiconazole $25 \%$ EC & $\begin{array}{c}00.00 \\
(00.00)\end{array}$ & $\begin{array}{c}00.00 \\
(00.00) \\
\end{array}$ & $\begin{array}{c}00.00 \\
(00.00)\end{array}$ & $\begin{array}{c}00.00 \\
(00.00)\end{array}$ & $100(90.00)$ & $\begin{array}{c}100 \\
(90.00)\end{array}$ & $\begin{array}{c}100 \\
(90.00)\end{array}$ & $\begin{array}{c}100 \\
(90.00)\end{array}$ \\
\hline $\mathrm{T}_{2}$ & Hexaconazole $05 \%$ EC & $00.00(00.00)$ & $\begin{array}{c}00.00 \\
(00.00)\end{array}$ & $\begin{array}{c}00.00 \\
(00.00)\end{array}$ & $\begin{array}{c}00.00 \\
(00.00)\end{array}$ & $100(90.00)$ & $\begin{array}{c}100 \\
(90.00)\end{array}$ & $\begin{array}{c}100 \\
(90.00)\end{array}$ & $\begin{array}{c}100 \\
(90.00)\end{array}$ \\
\hline $\mathrm{T}_{3}$ & Difenconazole $25 \%$ EC & $\begin{array}{c}13.34 \\
(21.42)\end{array}$ & $\begin{array}{c}10.70 \\
(19.09)\end{array}$ & $\begin{array}{c}8.86 \\
(17.31) \\
\end{array}$ & $\begin{array}{c}10.96 \\
(19.33)\end{array}$ & $\begin{array}{c}85.17 \\
(67.35)\end{array}$ & $\begin{array}{c}88.11 \\
(69.82)\end{array}$ & $\begin{array}{c}90.15 \\
(71.70)\end{array}$ & $\begin{array}{c}87.81 \\
(69.56)\end{array}$ \\
\hline $\mathrm{T}_{4}$ & Penconazole $10 \%$ EC & $\begin{array}{c}13.59 \\
(21.63)\end{array}$ & $\begin{array}{c}13.37 \\
(21.44)\end{array}$ & $\begin{array}{c}10.48 \\
(18.88)\end{array}$ & $\begin{array}{c}12.48 \\
(20.68)\end{array}$ & $\begin{array}{c}84.90 \\
(67.13)\end{array}$ & $\begin{array}{c}85.14 \\
(67.32)\end{array}$ & $\begin{array}{c}88.35 \\
(70.04)\end{array}$ & $\begin{array}{c}86.13 \\
(68.13)\end{array}$ \\
\hline $\mathrm{T}_{5}$ & $\begin{array}{c}\text { Thiophanate methyl } \\
\text { 70\% WP }\end{array}$ & $\begin{array}{c}65.20 \\
(53.84)\end{array}$ & $\begin{array}{c}58.39 \\
(49.82)\end{array}$ & $\begin{array}{c}50.92 \\
(45.52)\end{array}$ & $\begin{array}{c}58.15 \\
(49.69)\end{array}$ & $\begin{array}{c}27.55 \\
(31.66)\end{array}$ & $\begin{array}{c}35.12 \\
(36.34)\end{array}$ & $\begin{array}{c}43.40 \\
(41.20)\end{array}$ & $\begin{array}{c}35.35 \\
(36.48)\end{array}$ \\
\hline $\mathrm{T}_{6}$ & Carbendazim 50\% WP & $\begin{array}{c}52.90 \\
(46.66)\end{array}$ & $\begin{array}{c}47.52 \\
(43.57) \\
\end{array}$ & $\begin{array}{c}40.45 \\
(39.49) \\
\end{array}$ & $\begin{array}{c}46.95 \\
(43.25)\end{array}$ & $\begin{array}{c}41.22 \\
(39.94)\end{array}$ & $\begin{array}{c}47.20 \\
(43.39) \\
\end{array}$ & $\begin{array}{c}55.05 \\
(47.89) \\
\end{array}$ & $\begin{array}{c}47.82 \\
(43.75) \\
\end{array}$ \\
\hline $\mathrm{T}_{7}$ & Control (untreated) & $\begin{array}{l}90.00 \\
(71.56) \\
\end{array}$ & $\begin{array}{c}90.00 \\
(71.56) \\
\end{array}$ & $\begin{array}{c}90.00 \\
(71.56) \\
\end{array}$ & $\begin{array}{l}90.00 \\
(71.56) \\
\end{array}$ & $\begin{array}{c}0.00 \\
(0.00)\end{array}$ & $\begin{array}{c}0.00 \\
(0.00) \\
\end{array}$ & $\begin{array}{c}0.00 \\
(0.00) \\
\end{array}$ & $\begin{array}{c}0.00 \\
(0.00)\end{array}$ \\
\hline & $\mathrm{SE} \pm$ & 0.44 & 0.49 & 0.48 & - & 0.51 & 0.38 & 0.44 & - \\
\hline & $\mathrm{CD}(\mathrm{P}=0.01)$ & 1.36 & 1.51 & 1.48 & - & 1.56 & 1.19 & 1.36 & - \\
\hline
\end{tabular}
with untreated control.

Table 1: In vitro efficacy of systemic fungicides against $A$. alternata, causing chrysanthemum blight

Thus, all of the six systemic fungicides tested were found anti-causal against $A$. alternata and significantly inhibited its mycelial growth. However, fungicides found most effective in their order of merit were, Propiconazole 25\% EC and Hexaconazole 5\% EC> Difenconazole 25\% EC> Penconazole 10 EC> Carbendazim 50\% WP and Thiophanate methyl 70\% WP.
These results are in conformity to the reports of several earlier workers. Fungicides, Propiconazole 25\% EC and Hexaconazole 5\% EC were reported to cause maximum mycelial growth inhibition in many Alternaria spp. (Arunkumar and Kamanna, 2009; Apet et al., 2014; Thejakumar and Devappa 2016; Bhat et al., 2017; Shindhe et 
al., 2018; Vijayalakshmi et al., 2018; Mahadevaswamy et al., 2019).

Mycelial growth inhibition of non-systemic and two combi-product fungicides: All five non-systemic and two combi-product fungicides evaluated in vitro (each @ 1000, 2000 and $2500 \mathrm{ppm}$ ) were found effective in mycelia growth inhibition of A. alternata (PLATE-II, Table 2 and Fig. 2.) and it was increased with increasing concentrations of the test pathogen. Results (PLATE II, Table 2, Fig- 2) indicated that, non systemic and combi-product tested (each @ 1000, 2000 and $2500 \mathrm{ppm}$ ) significantly inhibited mycelial growth of $A$. alternata in the range of 48.50 to 78.60 per cent, 49.45 to
82.04 per cent and 58.08 to 85.25 per cent, respectively @ 1000, 2000 and 2500 ppm over untreated control and it was directly, proportional to the concentration of the fungicides tested. Average mycelial growth inhibition with the test fungicides ranged from 52.01 to 81.96 per cent. The fungicide, Carbendazim 12\% WP + Mancozeb 63\% WP showed highest growth inhibition (81.96\%). It was followed by Mancozeb 75\% WP (78.05\%), Copperoxy chloride 50\% WP $(69.36 \%)$, Copper hydroxide $77 \%$ WP (67.22\%), Propineb 70\% WP (58.65\%), Metalaxyl 8\% + Mancozeb 64\% WP (56.93\%) and Chlorothalonil $75 \%$ WP (52.01\%), respectively.

Table 2: In vitro efficacy of non systemic and combi-product fungicides

\begin{tabular}{|c|c|c|c|c|c|c|c|c|c|}
\hline \multirow{2}{*}{$\begin{array}{l}\text { Tr. } \\
\text { No. }\end{array}$} & \multirow{2}{*}{ Treatments } & \multicolumn{3}{|c|}{ Col. Dia.* (mm) at ppm } & \multirow{2}{*}{ Av. (mm) } & \multicolumn{3}{|c|}{ \% Inhibition* at ppm } & \multirow{2}{*}{$\begin{array}{c}\text { Av. Inhib. } \\
(\%)\end{array}$} \\
\hline & & 1000 & 2000 & 2500 & & 1000 & 2000 & 2500 & \\
\hline $\mathrm{T}_{1}$ & Chlorothalonil 75\% WP & $\begin{array}{c}46.35 \\
(42.90) \\
\end{array}$ & $\begin{array}{c}45.49 \\
(42.41) \\
\end{array}$ & $\begin{array}{c}37.72 \\
(37.89)\end{array}$ & $\begin{array}{c}43.18 \\
(41.08)\end{array}$ & $\begin{array}{c}48.50 \\
(44.14) \\
\end{array}$ & $\begin{array}{c}49.45 \\
(44.68) \\
\end{array}$ & $\begin{array}{c}58.08 \\
(49.64)\end{array}$ & $\begin{array}{c}52.01 \\
(46.15)\end{array}$ \\
\hline $\mathrm{T}_{2}$ & $\begin{array}{c}\text { Metalaxyl 8\% + } \\
\text { Mancozeb 64\% WP }\end{array}$ & $\begin{array}{c}40.27 \\
(39.38)\end{array}$ & $\begin{array}{c}39.28 \\
(38.80)\end{array}$ & $\begin{array}{c}36.72 \\
(37.29)\end{array}$ & $\begin{array}{c}38.75 \\
(38.49)\end{array}$ & $\begin{array}{c}55.25 \\
(48.01)\end{array}$ & $\begin{array}{c}56.35 \\
(48.64)\end{array}$ & $\begin{array}{c}59.20 \\
(50.30)\end{array}$ & $\begin{array}{c}56.93 \\
(48.98) \\
\end{array}$ \\
\hline $\mathrm{T}_{3}$ & $\begin{array}{c}\text { Carbendazim } 12 \mathrm{WP}+ \\
\text { Mancozeb } 63 \mathrm{WP}\end{array}$ & $\begin{array}{c}19.26 \\
(26.03)\end{array}$ & $\begin{array}{c}16.16 \\
(23.70)\end{array}$ & $\begin{array}{c}13.27 \\
(21.36)\end{array}$ & $\begin{array}{c}16.23 \\
(23.75)\end{array}$ & $\begin{array}{l}78.60 \\
(62.44)\end{array}$ & $\begin{array}{c}82.04 \\
(64.92) \\
\end{array}$ & $\begin{array}{c}85.25 \\
(67.41)\end{array}$ & $\begin{array}{c}81.96 \\
(64.86)\end{array}$ \\
\hline $\mathrm{T}_{4}$ & $\begin{array}{c}\text { Copperoxy chloride } \\
50 \% \text { WP }\end{array}$ & $\begin{array}{c}31.46 \\
(34.11) \\
\end{array}$ & $\begin{array}{c}26.68 \\
(31.09)\end{array}$ & $\begin{array}{c}24.57 \\
(29.71)\end{array}$ & $\begin{array}{c}27.57 \\
(31.67)\end{array}$ & $\begin{array}{c}65.04 \\
(53.75)\end{array}$ & $\begin{array}{c}70.35 \\
(57.00)\end{array}$ & $\begin{array}{c}72.70 \\
(58.50)\end{array}$ & $\begin{array}{c}69.36 \\
(56.39)\end{array}$ \\
\hline $\mathrm{T}_{5}$ & $\begin{array}{c}\text { Copper-hydroxide } \\
77 \% \text { WP } \\
\end{array}$ & $\begin{array}{c}32.96 \\
(35.03) \\
\end{array}$ & $\begin{array}{c}28.26 \\
(32.11) \\
\end{array}$ & $\begin{array}{c}27.27 \\
(31.48) \\
\end{array}$ & $\begin{array}{c}29.49 \\
(32.89) \\
\end{array}$ & $\begin{array}{c}63.37 \\
(52.75) \\
\end{array}$ & $\begin{array}{r}68.60 \\
(55.91) \\
\end{array}$ & $\begin{array}{r}69.70 \\
(56.60) \\
\end{array}$ & $\begin{array}{c}67.22 \\
(55.07) \\
\end{array}$ \\
\hline $\mathrm{T}_{6}$ & $\begin{array}{l}\text { Mancozeb } \\
75 \% \text { WP }\end{array}$ & $\begin{array}{c}21.24 \\
(27.44)\end{array}$ & $\begin{array}{c}20.50 \\
(26.92)\end{array}$ & $\begin{array}{c}17.50 \\
(24.72)\end{array}$ & $\begin{array}{c}19.74 \\
(26.37)\end{array}$ & $\begin{array}{c}76.40 \\
(60.93)\end{array}$ & $\begin{array}{c}77.22 \\
(61.49) \\
\end{array}$ & $\begin{array}{c}80.55 \\
(63.83) \\
\end{array}$ & $\begin{array}{c}78.05 \\
(62.06)\end{array}$ \\
\hline $\mathrm{T}_{7}$ & $\begin{array}{l}\text { Propineb } \\
70 \% \text { WP }\end{array}$ & $\begin{array}{c}40.14 \\
(39.31)\end{array}$ & $\begin{array}{c}38.43 \\
(38.31)\end{array}$ & $\begin{array}{c}33.07 \\
(35.10)\end{array}$ & $\begin{array}{c}37.21 \\
(37.58)\end{array}$ & $\begin{array}{c}55.40 \\
(48.10)\end{array}$ & $\begin{array}{c}57.30 \\
(49.19)\end{array}$ & $\begin{array}{c}63.25 \\
(52.68)\end{array}$ & $\begin{array}{c}58.65 \\
(49.98)\end{array}$ \\
\hline $\mathrm{T}_{8}$ & Control (untreated) & $\begin{array}{c}90.00 \\
(71.56) \\
\end{array}$ & $\begin{array}{c}90.00 \\
(71.56) \\
\end{array}$ & $\begin{array}{c}90.00 \\
(71.56) \\
\end{array}$ & $\begin{array}{l}90.00 \\
(71.56) \\
\end{array}$ & $\begin{array}{c}0.00 \\
(0.00) \\
\end{array}$ & $\begin{array}{c}0.00 \\
(0.00) \\
\end{array}$ & $\begin{array}{c}0.00 \\
(0.00) \\
\end{array}$ & $\begin{array}{c}0.00 \\
(0.00)\end{array}$ \\
\hline & $\mathrm{SE} \pm$ & 0.55 & 0.73 & 0.59 & - & 0.55 & 0.55 & 0.62 & - \\
\hline & $\mathrm{CD}(\mathrm{P}=\mathbf{0 . 0 1})$ & 1.67 & 2.21 & 1.80 & - & 1.66 & 1.67 & 1.89 & - \\
\hline
\end{tabular}

* Mean of three replication

Figures in parentheses are arcsine transformed values

Thus, based on efficiency, fungicides in the order of merit were Carbendazim 12\% + Mancozeb 63\% WP> Mancozeb 75\% WP> Copperoxy chloride 50\% WP> Copper hydroxide $77 \%$ WP> Propineb 70\% WP> Metalaxyl 8\% + Mancozeb $64 \% \mathrm{WP}$ and Chlorothalonil $75 \% \mathrm{WP}$, respectively.

These results of the present study are in consonance with the findings of many earlier workers, who reported effectiveness of non-systemic fungicides such as Propineb $70 \% \mathrm{WP}$, Mancozeb75\% WP, Copperoxy chloride 50\% WP, Copper hydroxide $77 \%$ WP, Chlorothalonil $75 \%$ WP etc. against many phytopathogenic Alternaria spp. (Bavaji et al., 2012; ${ }^{5}$ Apet et al., 2014; Thejakumar and Devappa, 2016; Bhat et al., 2017; Shindhe et al., 2018; Vijayalakshmi et al., 2018; Mahadevaswamy et al., 2019), combi-product such as Carbendazim 12\% + Mancozeb 63\% WP, Metalaxyl 8\%+ Mancozeb 64\% WP against many phytopathogenic Alternaria spp. (Gholve et al., $2014^{[9]}$; Shamala and Janardhana, 2015) $[9,15]$.

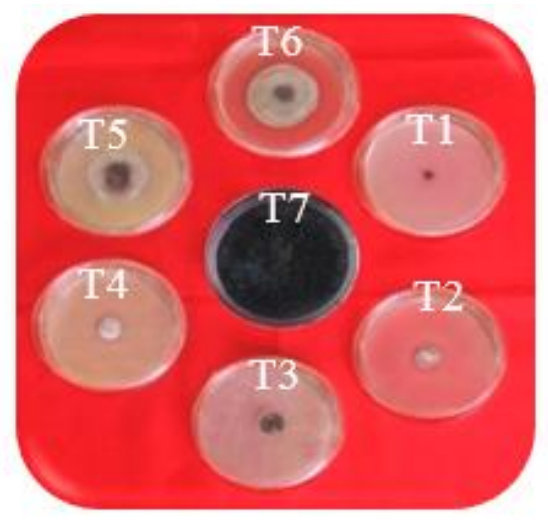

$500 \mathrm{ppm}$

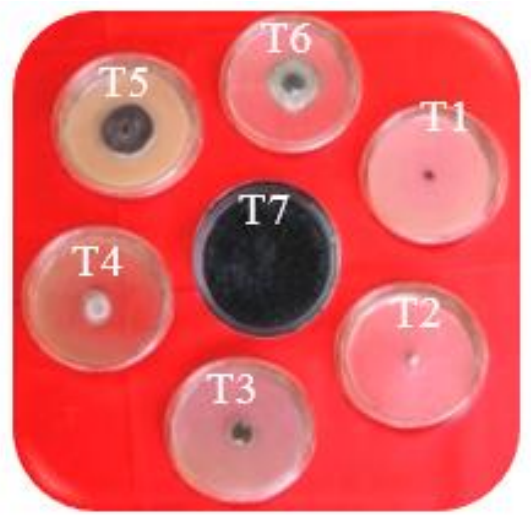

$1000 \mathrm{ppm}$

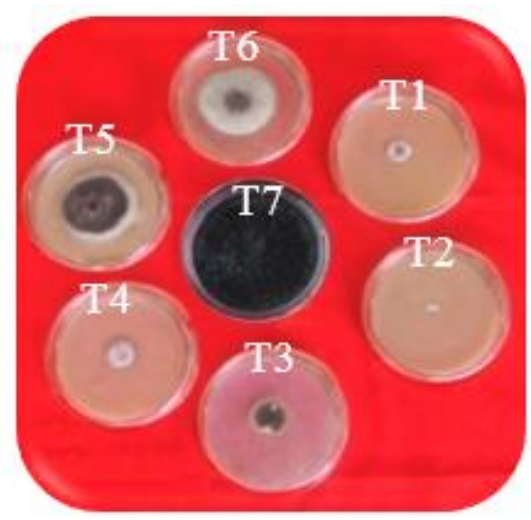

$1500 \mathrm{ppm}$

Plate I: In vitro efficacy of systemic fungicides against A. alternata, causing chrysanthemum blight 


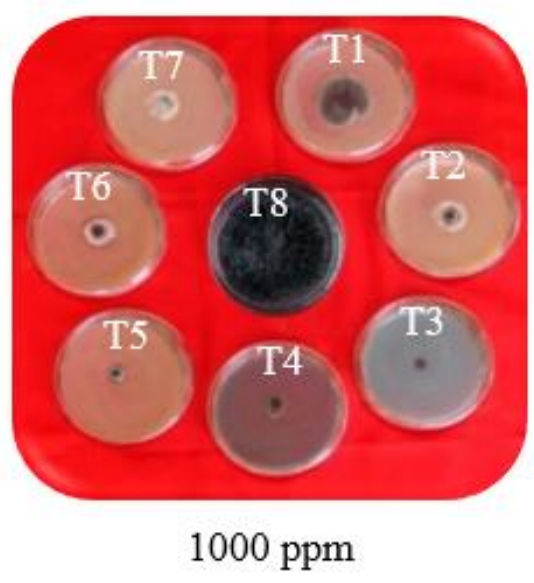

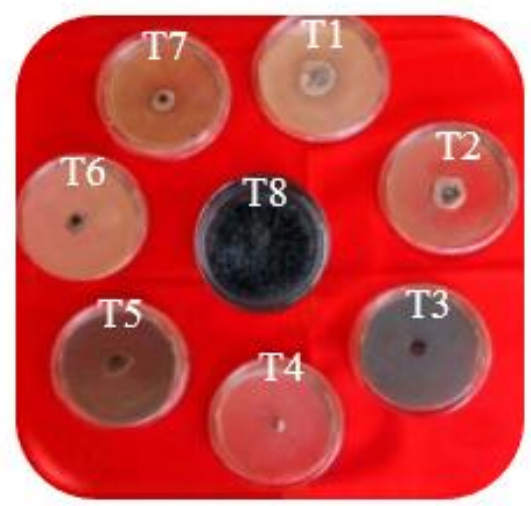

2000 ppm

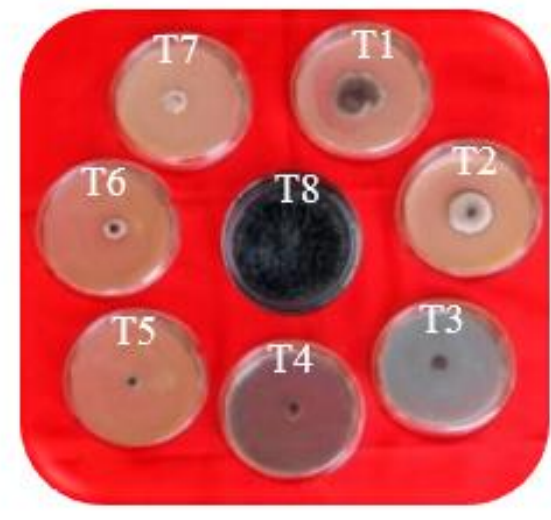

$2500 \mathrm{ppm}$

Plate II: In vitro efficacy of non- systemic / combi-product fungicides against A. alternata, causing chrysanthemum blight
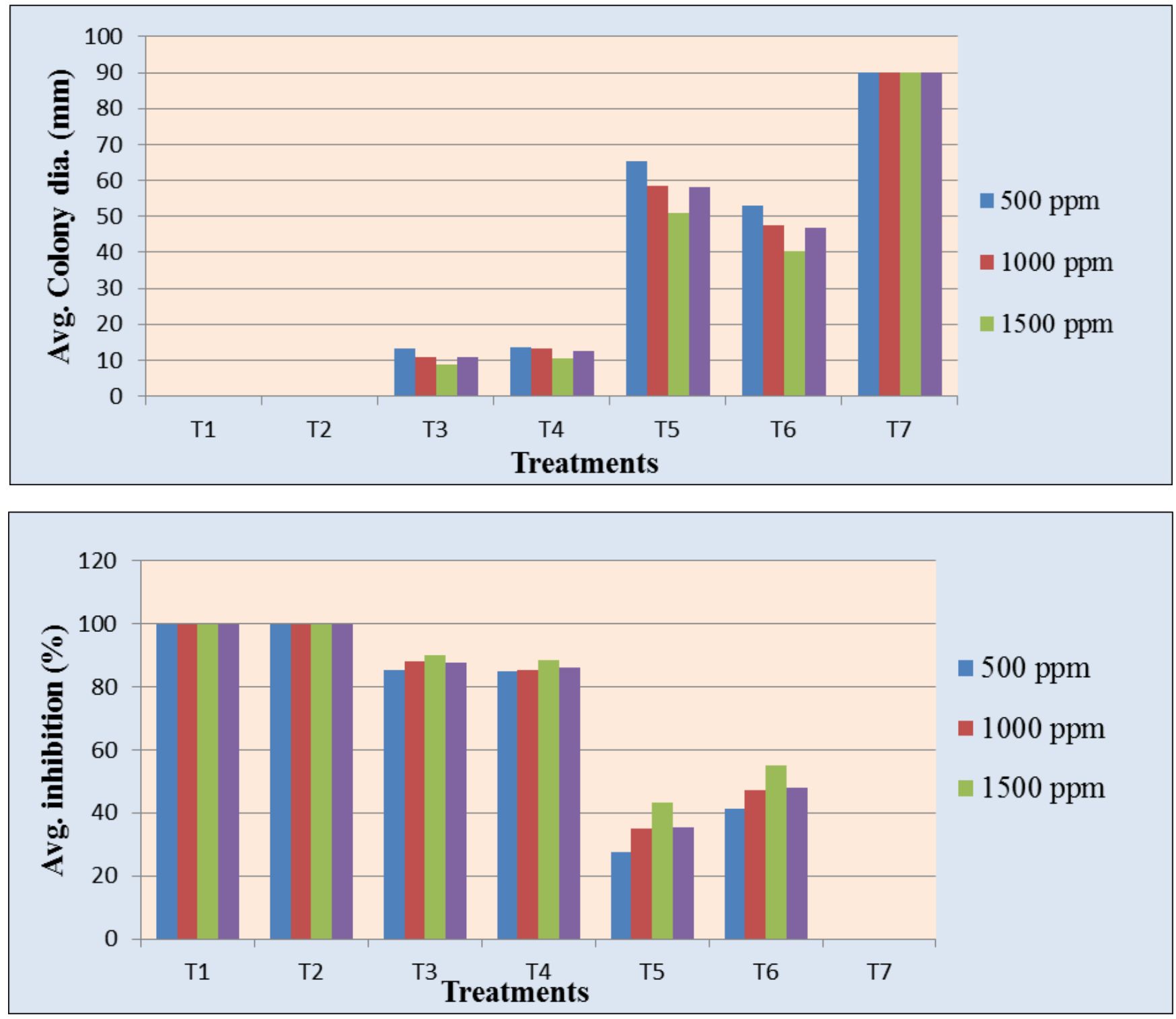

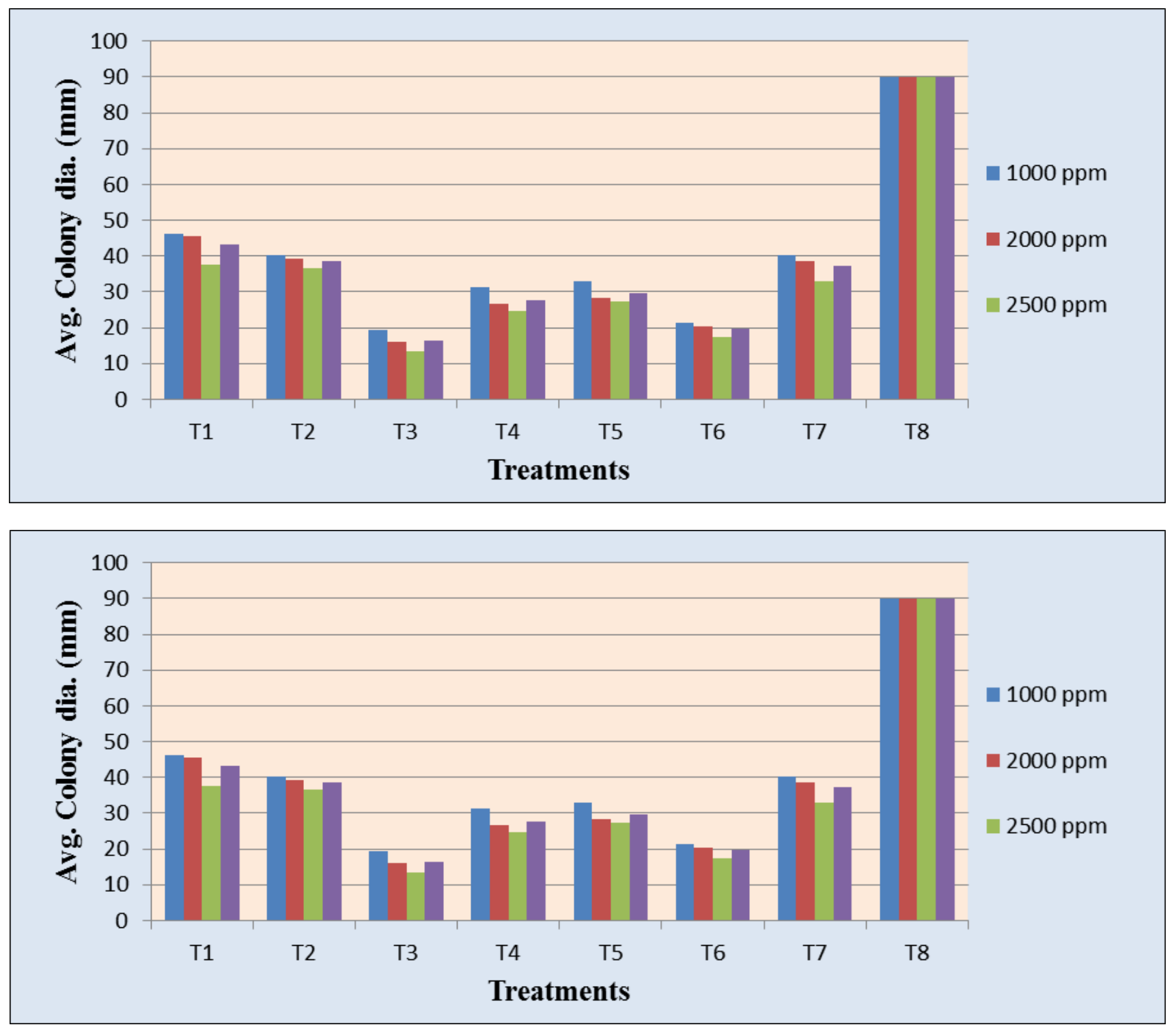

Fig 1: In vitro efficacy of systemic fungicides against $A$. alternata, causing chrysanthemum leaf blight

\section{Conclusion}

Hence, from ongoing results and discussion, it is concluded that, all of the seven systemic (each@ @ 500, 1000 and 1500 ppm) and five contact and two combi-product fungicides (@1000, 2000 and 2500 ppm) evaluated in vitro were found effective and fungistatic against $A$. alternata. However, Based on average mycelial growth inhibition, the systemic fungicides viz., Propiconazole 25\% EC, Hexaconazole 5\% EC, Difenconazole 25\% EC, Penconazole 10 EC, Carbendazim 50\% WP and Thiophanate methyl 70\% WP were found most effective against the test pathogen. Whereas, among contact / combi-product fungicides, the most effective fungicides found were Carbendazim 12\% + Mancozeb 63\% WP, Mancozeb 75\% WP, Copperoxy chloride 50\% WP, Copper hydroxide 77\% WP, Propineb 70\% WP, Metalaxyl $8 \%+$ Mancozeb 64\% WP and Chlorothalonil 75\% WP, respectively.

\section{Acknowledgment}

We are very much thankful to the Head of the Department of Plant Pathology, College of Agriculture, Latur for providing the laboratory facilities.

\section{References}

1. Anonymous. Area and Production of Horticultural Crops. WWW.Agricoop.nic.in., 2018.
2. Apet KT, Jagdale JS, Chavan PG, More AS, Mirza FN, Baig. In vitro evaluation of fungicides, botanicals and bio-agents against Alternaria alternata, causing leaf spot of gerbera. Trends Biosci., 2014;7(21):3374-3382.

3. Arunkumar GS. Studies on leaf blight of chrysanthemum caused by Alternaria alternata (Fr.) Keissler. M. Sc. (Agri.) Thesis, Univ. Agric. Sci., Dharwad., 2008.

4. Arunkumar GS, Kamanna BC. In vitro evaluation of fungicides, botanicals and bio- agents against Alternaria alternata (Fr.) Keissler causing leaf blight of chrysanthemum. Green Farming., 2009;2(12):868-870.

5. Bavaji M, Khamarjahan MD, Mahendra Nath M. In vitro evaluation of fungicide and plant extracts on the incidence of leaf blight on sesame caused by Alternaria alternata (Fr.) Keissler. Int. J. Food. Agric. Vet. Sci., 2012;2(3):105-107.

6. Bhat HA, Ahmad K, Ahanger RA, Wani SH, Bhat AH, Showket AD. Fungicidal management of leaf blight of gerbera (Gerbera jamesonii Hook) in Kashmir valley. J. Pharma. Phytochem., 2017;6(4):384-387.

7. Divyajyothi U, Ekabote Suresh D, Narayanswamy H, Narayanaswamy P, Ravindra H, Satish KM. Evaluation of new fungicide molecules against leaf blight of chrysanthemum caused by Alternaria spp. Int. J. Curr. Microbiol. App. Sci., 2018;7(7):3884-3890. 
8. Ellis MB. Dematiaceous hyphomycetes. Commonwealth Mycological Institute, Kew, Surry, England: 1971;465466:469-470.

9. Gholve VM, Jogdand SM, Suryawanshi AP. Evaluation of fungicides, botanicals and bioagents against Alternaria leaf blight caused by Alternaria macrospora in cotton. J. Cotton Res. Dev., 2014;28(2):327-331.

10. Kalia R. Effect of different concentrations of auxins on the regeneration of Chrysanthemum morifolium plantlets. Int. J. Tech. Res. Appli., 2015;3(6):106107.

11. Mahadevaswamy G, Vijayalakshmi G, Gangadharanaik $\mathrm{G}$. In vitro evaluation of fungicides, plant extracts and bio-agents against Alternaria helianthi, causing leaf blight of sunflower. Int. J. Curr. Microbiol. App. Sci., 2019;8(2):1632-1639.

12. Nene YL, Thapliyal PN. Evaluation of fungicides In: Plant Disease Control (3" ed.) Oxford, IBH Pub. Co. Pvt. Ltd., New Delhi., 1993, 531-532.

13. Panse VV, Sukhatme PV. Statstical methods for Agricultural worker. ICAR publication 3 rd: 1978, 157165.

14. Pradeepkumar T, Sum B, Jyotibhaskar, Satheesan KN. Management of Horticultural crops. Pub. by IARI, New Delhi. : 2008, 440.

15. Shamala G, Janardhana GR. Prevalence and severity of leaf blight disease of chrysanthemum in southern regions of Karnataka and in-vitro fungicidal effect on Alternaria alternata. Int. J. Life Sci., 2015;9(3):38-45.

16. Shindhe Mahadev, Narayanaswamy H, Murali R, Nagaraja $H$. Efficacy of different fungicides against Alternaria tagetica, causing leaf spot and flower blight of marigold under in vitro condition. Int. J. Curr. Microbiol. App. Sci., 2018;7(6):2251-2255.

17. Thejakumar MB, Devappa V. Efficacy of different fungicides against Alternaria alternata and Cercospora capsici under in-vitro conditions. Int. J. Adv. Res. Biol. Sci., 2016;3(5):126-129.

18. Tupe SB, Perke DS, Korade VC. Costs, returns and profitability of chrysanthemum in Ahmednagar district of Maharashtra State.Agric. Update, 2017;12(TECHSEAR2):389-392.

19. Tuite J. Plant pathological methods. Fungi and Bacteria. Minneapolis, Minnesota, USA. Burgess Publishing Company, 1969,239.

20. Vijayalakshmi G, Karuna K, Mahadevaswamy G. Evaluation of microbial bio-control agents and fungicides against Alternaria helianthi, causing leaf blight of sunflower. Int. J. Curr. Microbiol. App. Sci., 2018;7(01):2726-2730.

21. Vincent JM. Distortion of fungal hyphae in the presence of certain inhibitors. Nature, 1927, 159-180. 